\title{
The 3D printing of dielectric elastomer films assisted by electrostatic
}

\section{force}

Yuhao Wang ${ }^{1}$, Yanfen Zhou ${ }^{1,2}$, Wenyue Li $^{1}$, Zhanxu Liu ${ }^{1}$, Bangze Zhou ${ }^{1}$, Shipeng Wen ${ }^{3}$, Liang Jiang ${ }^{1,2,{ }^{*}}$, Shaojuan Chen ${ }^{1,2}$, Jianwei Ma ${ }^{1,2}$, Anthony Betts ${ }^{4}$, Stephen Jerrams ${ }^{4}$, Fenglei Zhou ${ }^{1,5}$

${ }^{1}$ College of Textiles and Clothing, Qingdao University, Qingdao, 266071, China

${ }^{2}$ Eco-Textile Collaborative Innovation Center, Qingdao University, Qingdao, 266071, China

${ }^{3}$ Beijing Engineering Research Center of Advanced Elastomers, Beijing University of Chemical Technology, Beijing 100029, China

${ }^{4}$ Technological University Dublin (TUD), City Campus, Kevin St, Dublin D08 NF82, Ireland

${ }^{5}$ Centre for Medical Image Computing, University College London, London, WC1V $6 \mathrm{LJ}, \mathrm{UK}$

\begin{abstract}
:
Compared with traditional methods for preparing dielectric elastomer (DE) films, electrohydrodynamic (EHD) 3D printing displays many advantages, notably full automation, computer control and flexible design. It also confers high printing resolution, high preparation efficiency with minimal probability of nozzle clogging. In this article, EHD 3D printing was employed to fabricate silicone rubber (SR) based DE films. In order to increase their dielectric constant, high dielectric copper phthalocyanine $(\mathrm{CuPc})$ particles were added into the SR ink. Optimal printing
\end{abstract}

\footnotetext{
${ }^{*}$ Corresponding author, email address: liang.jiang@qdu.edu.cn
} 
conditions were determined by analyzing the effects of printing voltage and ink properties on the formation of liquid cone and the printed line width. The SR/CuPc composite film with $3 \mathrm{wt} \% \mathrm{CuPc}$ particles (SR/CuPc-3) exhibits a high dielectric constant of 5.52, with a large actuated area strain of $23.7 \%$ under an electric field of $39.4 \mathrm{~V} / \mu \mathrm{m}$. Furthermore, under 100 cycles of electric field loading, SR/CuPc-3 demonstrate excellent electromechanical stability, indicating that EHD 3D printing holds a considerable potential for fabricating high-performance DE films in an efficacious manner.

Keywords: Electrohydrodynamic 3D printing; Dielectric elastomer; Silicone rubber; Copper phthalocyanine

\section{Introduction}

Dielectric elastomers (DEs) are a type of smart material that can produce large electromechanical actuation under electrical stimulus [1-3]. Because DEs can undergo large deformations and exhibit light weight, rapid response and high energy density, they are considered to be good candidates for applications in the fields of micro-robotics, artificial intelligence, biomimetics and sensors $[4,5]$. A DE sandwiched between two complaint electrodes can produce an expansion in area and a reduction in thickness, when an electric filed is applied. The actuated strain $\left(s_{z}\right)$ of a DE is determined by the following equation [6]:

$$
s_{z}=-\frac{p}{Y}=-\frac{\varepsilon_{0} \varepsilon_{r} E^{2}}{Y}
$$

where $p$ is the Maxwell stress, $Y$ is Young's (elastic) modulus, $\varepsilon_{0}$ and $\varepsilon_{r}$ are the permittivity of vacuum $\left(8.85 \times 10^{-12} \mathrm{~F} / \mathrm{m}\right)$ and the dielectric constant of the DE respectively and $E$ is the electric field strength. This equation indicates that a low modulus and a high dielectric constant are beneficial for generating large actuated strains $[7,8]$. However, a low modulus will also often result in poor mechanical 
properties of the resultant materials, eventually weakening their long-term performance [9]. The dielectric constant of a DE can be enhanced by blending the material with high permittivity ceramics, such as barium titanate $\left(\mathrm{BaTiO}_{3}\right)$ [10-12], lead magnesium niobatelead titanate (PMN-PT) [13], $\mathrm{Ba}_{0.6} \mathrm{Sr}_{0.4} \mathrm{TiO}_{3}[14,15]$ or high permittivity metallo-organic semiconductors, such as copper phthalocyanine $(\mathrm{CuPc})[16]$. Thus, the actuated strain of a DE can be significantly improved via this blending based approach. The reported techniques used to prepare DE films include blade coating [17], spin coating [18] and spray deposition [19]. Compared to these traditional methods, a novel method, utilizing three-dimensional (3D) printing to prepare DE films, offers the additional advantages of full automation, computed control, low production costs and flexible design $[20,21]$. Therefore, 3D printing holds great promise for fabricating excellent DE films. Mccoul et al. [22] used inkjet 3D printing to prepare DE actuator (DEA) devices. The resultant high-quality printed silicone elastomer membranes, with thicknesses of $2 \mu \mathrm{m}$, exhibited a maximum tensile strain of $727 \%$ and an actuated area strain of up to $2.4 \%$ at breakdown strength of $130 \mathrm{~V} / \mu \mathrm{m}$. By comparison, the performance of the inkjet 3D printed silicone membrane was almost the same as that of a blade-casted silicone elastomer membrane. However, the inkjet 3D printing method is subject to easy nozzle clogging and low preparation efficiency.

Electrohydrodynamic (EHD) 3D printing, as a newly emerging high-resolution printing approach, has attracted increasing interest because of its capacity of direct-writing 3D structures. The mechanism behind EHD printing is to apply an electric field formed between a nozzle and a conductive substrate onto an electrically charged liquid droplet. Then liquid droplet extruded from the nozzle tip under the influence of an electric field force breaks out into a fine jet, whose size is invariably much smaller than the nozzle $[23,24]$. EHD 3D printing can overcome the above-mentioned drawbacks of inkjet 
printing with obtaining the advantages of high efficiency and minimized probability of clogging the nozzle $[25,26]$, which provide greater possibilities for EHD 3D printing to prepare film materials.

In this work, EHD 3D printing technique was utilized to prepare the silicone rubber (SR) DE films. In order to improve the electromechanical performance of the SR, high dielectric $\mathrm{CuPc}$ particles were adopted as fillers and added into pure SR. The evolution of the liquid cone and jet resulting from applied high voltages and variations in $\mathrm{CuPc}$ content during EHD printing was monitored by a high-speed camera, with the aim of obtaining a high printing resolution. In addition, the mechanical and electromechanical properties of the printed SR/CuPc composite film were also investigated in an attempt to ascertain their characteristic behavior and confirm the benefit of using the $\mathrm{CuPc}$ in the ink formulation.

\section{Experimentation}

\subsection{Materials}

Two-component silicone LSR 4305 was purchased from Bluestar Ltd., USA. CuPc was acquired from Aladdin Reagent Co., Ltd., Shanghai, China. N-heptane and toluene were all procured from Sinopharm Chemical Reagent Co., Ltd., China. The commercially available conductive carbon grease NYOGEL $756 \mathrm{G}$, which was used as the compliant electrode in this work, was provided by Nye Lubricants, Inc., Fairhaven, MA, USA.

\subsection{Preparation of pure SR and SR/CuPc inks}

The pure SR ink with a concentration of 30 wt.\% was prepared by mixing two components SR at a weight ratio of 1:1 and then dissolving in n-heptane.

The preparation of SR/CuPc ink was carried out by adding different weight contents of CuPc particles (1 wt.\%, 2 wt.\% and 3 wt.\%) into n-heptane firstly followed by sonication for $5 \mathrm{~min}$. Then two components SR at a weight ratio of 1:1 was mixed and 
dissolved in $\mathrm{CuPc} / \mathrm{n}$-heptane. The resulting SR/CuPc ink containing $1 \mathrm{wt} \%, 2 \mathrm{wt} \%$ and $3 \mathrm{wt} \%$ of CuPc particles were denoted as SR/CuPc-1 ink, SR/CuPc-2 ink and SR/CuPc3 ink respectively.

\subsection{EHD 3D printing of DE films}

Figure 1 is diagrammatic representation of the EHD 3D printing system consisting of a high voltage power supply (HPSN 1220, LANYI, Zhejiang, China), an ink supply, an ultrasonic device, a high-speed camera (WP-UT500, Shenzhen, China) and a conductive substrate fixed on a three-axis mechanical stage, which is programmed by the computer to move in the $X, Y$ and $Z$ mutually perpendicular axes. The high voltage supply was connected to an electrically-grounded conductive substrate and a nozzle with a blunt end, the outer diameter of which is $400 \mu \mathrm{m}$. The ink in the nozzle was supplied by a syringe pump (Longer Precision Pump Co., Ltd., Baoding, Hebei, China) operating at a typical flow rate of $0.07 \mu \mathrm{L} / \mathrm{min}$. During printing, ultrasonication was used to disperse particles to prevent the formation of precipitates and particle agglomeration. Finally, after finishing the printing process, the film was left into an oven, curing at $120^{\circ} \mathrm{C}$ for $1 \mathrm{~h}$ to ensure a complete evaporation of $\mathrm{n}$-heptane. EHD 3D printing was initiated by slowly increasing the nozzle voltage, until the pendant ink drops elongated and fell on to the printing substrate beneath. The morphology of the liquid cone was recorded by the high-speed camera. 


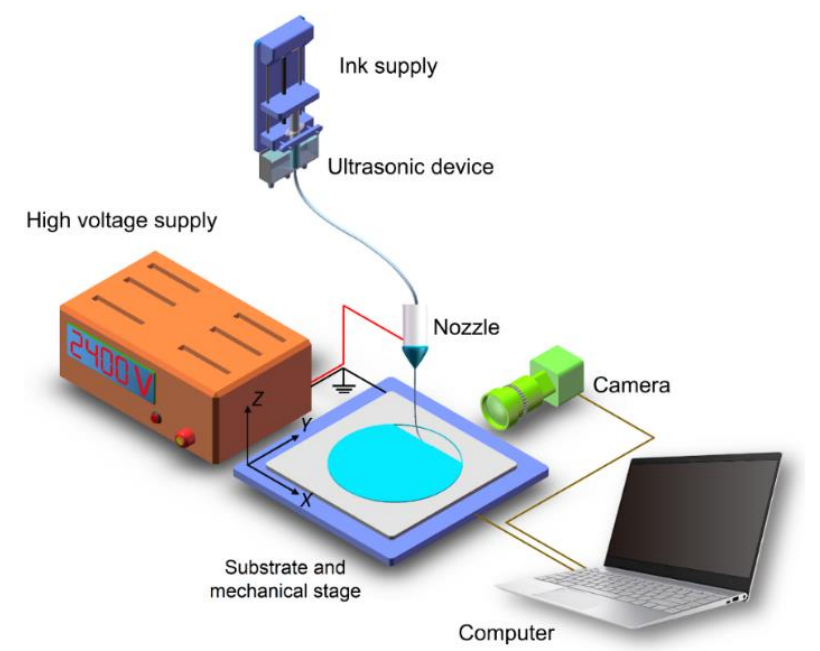

Figure 1 Schematic diagram showing a self-built EHD 3D printing system for fabrication of DE films.

\subsection{Characterization}

The viscosity of the inks was measured by using a rotating viscosity tester (NDJ-9s, Ping Xuan scientific instrument co., Ltd., China) at a rotational speed of $6 \mathrm{rpm}$.

The morphologies of the printed DE membranes based on SR filled with CuPc particles were observed by using a scanning electron microscope (SEM) (VEGA3, TESCAN, Czech Republic) which was equipped with an EDX (E1856-C2B, EDAX, USA).

The three-dimensional surface morphologies of the printed films were observed by using an ultra-depth 3D digital microscope (DVM6 M, Leica Microsystems Ltd., Germany).

Tensile tests were performed on the printed SR and SR/CuPc composite membranes using a universal tensile testing machine (Instron 5965 Tensile Machine, Glenview, US). The shapes of the specimens were printed according to the GB/T 528-2009 test standard. The crosshead speed during the tensile testing was $100 \mathrm{~mm} / \mathrm{min}$. The elastic moduli were determined from the slopes of the stress-strain curves using linear fits to the data points obtained within the 0 to $10 \%$ strain range.

The crosslink densitiy $n$ were determined by performing swelling tests in toluene at room temperature and applying the Flory-Rehner equation [27] 
$n=\frac{-\left[\ln \left(1-v_{2}\right)+v_{2}+\chi v_{2}{ }^{2}\right]}{v_{1}\left[v_{2}^{1 / 3}-v_{2} / 2\right]}$

where $v_{2}$ is the volume fraction of the film, $\chi$ with a value of 0.37 in this work is the interaction parameter between toluene and SR, $v_{l}$ is the molar volume of the toluene.

Dielectric measurements of SR and SR/CuPc composite films were conducted using a broadband dielectric spectrometer (E4990A, Keysight, USA) at $20{ }^{\circ} \mathrm{C}$ in the frequency range of $10 \mathrm{~Hz}$ to $10 \mathrm{MHz}$. The films of approximately $0.4 \mathrm{~mm}$ thickness were placed on a cell between gold-plated flat electrodes each of diameter of $20 \mathrm{~mm}$ and a thickness of $2 \mathrm{~mm}$.

The actuated area strain tests were performed by using an electromechanical testing system. The tests were executed using EHD 3D printed disc DE films with a diameter of $60 \mathrm{~mm}$ and a thickness of approximately $0.4 \mathrm{~mm}$ at ambient conditions. The DE films were initially pre-stretched equibiaxially to a ratio of 2.0 , then fixed on a circular sample clamp. Before each test, a compliant electrode with a diameter of $12 \mathrm{~mm}$ was coated on the samples for both sides of the sample. The electric field was incrementally applied with the voltage rising by $200 \mathrm{~V}$ per $3 \mathrm{~s}$ until electric breakdown occurred.

Each experimental data point is the average of the results obtained from at least three separate samples investigated under the same conditions.

\section{Results and discussion}

In the printing process, the ink droplet at the tip of the nozzle was deformed and stretched by electrostatic force produced between the nozzle and the conductive substrate when the voltage was applied, forming a liquid cone, whose apex emerged a thin jet. In detail, it can be envisaged from the force diagram of Figure 2(a) that the cone-jet mode formed only when the electrostatic force $\left(F_{e s}\right)$ was greater than force derived from ink viscosity $(\mu)$ plus surface tension $\left(F_{s t}\right)$ [28]. Under the coupling effect 
of electrostatic force, viscosity, surface tension, liquid pressure $\left(P_{l}\right)$ and air pressure $\left(P_{a}\right)$, the liquid cone was able to maintain its stability, resulting in the formation of highly desired cone-jet mode [29].

Before using EHD 3D printing to fabricate the designed structures, the optimization of ink properties and process parameters are indispensable procedures. Generally, for a specific ink, cone angle and jet diameter are two important criteria for evaluating the jet evolution with the increasing applied voltage. The properties of the ink, such as viscosity, also have great influence on the cone-jet mode and the printed structure, which were studied in this work.

(a)
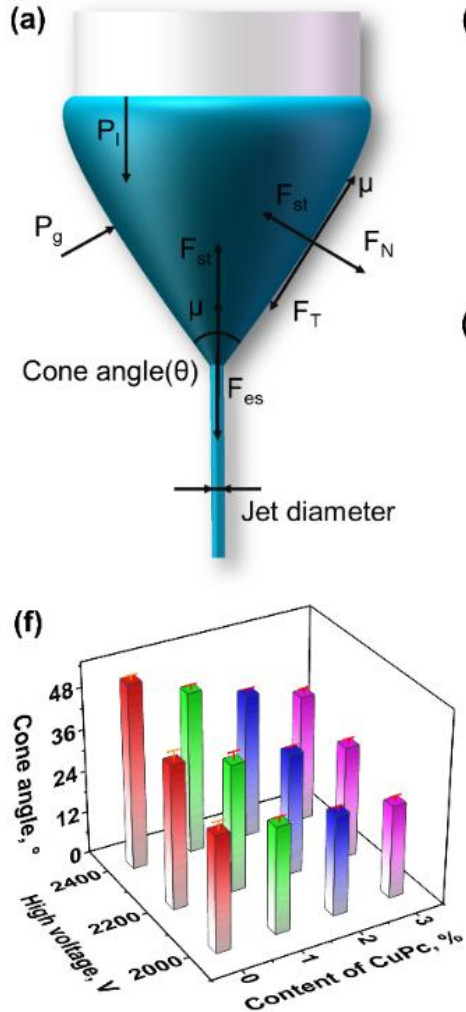

(b)

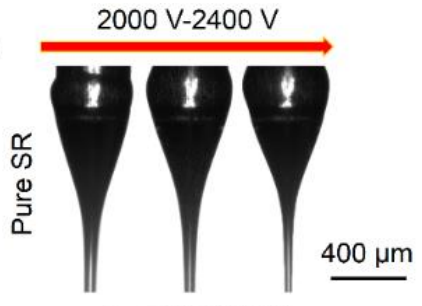

(d)
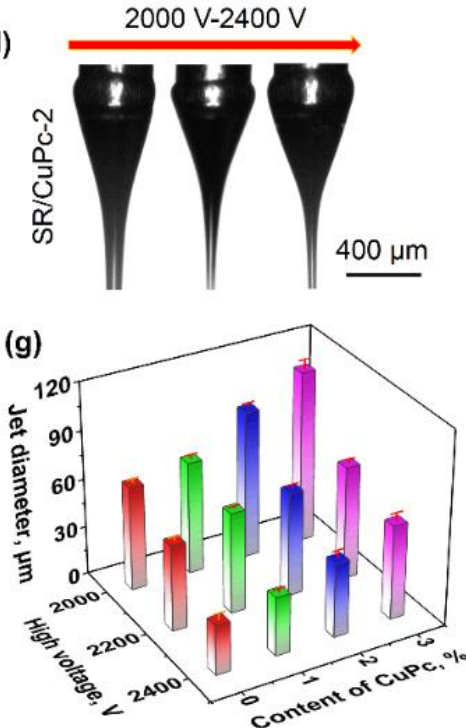

(c)

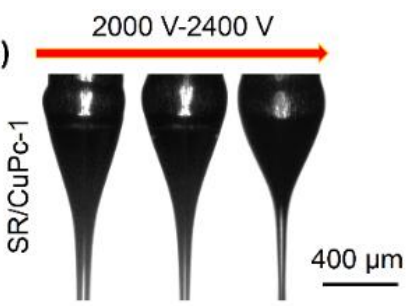

(e)
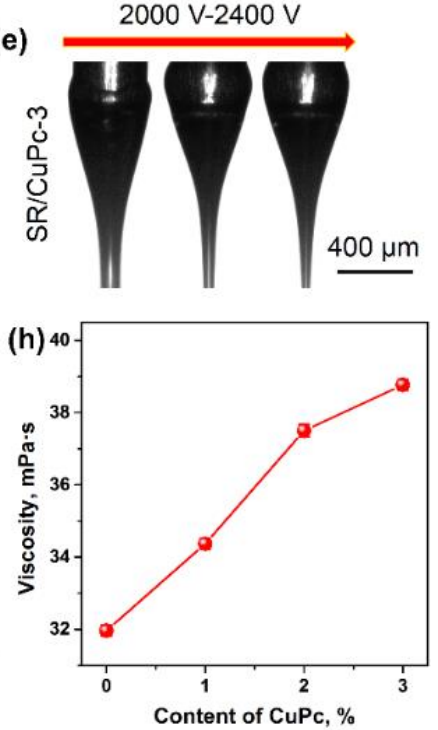

Figure 2 (a) Force diagram of the liquid cone; Jet behavior of the ink (b) pure SR, (c)

SR/CuPc-1, (d) SR/CuPc-2, (e) SR/CuPc-3 under high voltages of $2000 \mathrm{~V}$ to $2400 \mathrm{~V}$;

(f) Histogram of cone angle of SR/CuPc ink related to high voltage and content of

$\mathrm{CuPc}$; (g) Histogram of jet diameter of SR/CuPc ink related to high voltage and content of $\mathrm{CuPc}$; (h) Plot of viscosity as a function of $\mathrm{CuPc}$ content. 
As shown in Figures 2 (b-e), under different applied voltages, the conical shape of the ink containing various amount of CuPc particles deforms regularly. The cone angle of the liquid cone increases, for instance, the cone angle of the SR/CuPc-3 ink increases from $26 \pm 2^{\circ}$ to $36 \pm 2^{\circ}$ as the printed voltage increases from $2000 \mathrm{~V}$ to $2400 \mathrm{~V}$, which is depicted in Figure 2(f). It is expected that when the ink feeding rate remains constant, higher electrostatic force generated by the higher applied voltage will accelerate the flow speed at which the jet leaves the nozzle, thereby causing the conical droplet recede and consequently an increase in the cone angle [30]. As demonstrated in Figure 2(g), it is clear that the jet diameter, which was measured at $800 \mu \mathrm{m}$ below the nozzle tip, decreases as the printed voltage increases. Take the SR/CuPc ink containing 3 wt\% $\mathrm{CuPc}$ as an example, the jet diameter decreases remarkably from $107 \pm 6 \mu \mathrm{m}$ to $58 \pm 5$ $\mu \mathrm{m}$ when the voltage increases from $2000 \mathrm{~V}$ to $2400 \mathrm{~V}$. This phenomenon can be explained by using the force diagram shown in Figure 2(a). Under the applied voltage, the tangential electrostatic force $\left(F_{T}\right)$ at the interface of the ink droplet causes an axisymmetric convectional flow. Therefore, the ink at the interface moved more rapidly than the ink inside, resulting in a backflow at the center of the liquid cone [29]. The higher the applied voltage, the greater the electrostatic force and the stronger the backflow, resulting in a smaller jet diameter [31]. Furthermore, with an increase in the content of $\mathrm{CuPc}$ particles, the cone angle also decreases, while the jet diameter increases, as shown in Figures 2(f) and (g). For the voltage of $2400 \mathrm{~V}$, the cone angle decreases from $53 \pm 1^{\circ}$ to $36 \pm 2^{\circ}$, while the jet diameter increases from $31 \pm 4 \mu \mathrm{m}$ to $58 \pm 5 \mu \mathrm{m}$ when the $\mathrm{CuPc}$ content increases from 0 to $3 \mathrm{wt} \%$. As depicted in Figure 2(h), the viscosity of the ink increases from $31.97 \pm 0.15 \mathrm{mPa} \cdot \mathrm{s}$ to $38.77 \pm 0.15 \mathrm{mPa} \cdot \mathrm{s}$ as the content of $\mathrm{CuPc}$ particles increases from 0 to $3 \mathrm{wt} \%$. Due to the poor fluidity caused by the high viscosity [32], the jet flow speed is decelerated, resulting in a longer cone and 
the decreased cone angle. In addition, the high viscosity hinders the stretching of the ink during the printing process, resulting in a larger jet diameter. This phenomenon was also observed in previous studies [33, 34].

Figure 3(a) shows the schematic of EHD printing process of linear patterns. The width of the printed lines was measured and used to evaluate the printing resolution. As shown in Figures 3(b-e), as the content of CuPc particles added into the ink increases from 0 to $3 \mathrm{wt} \%$, the color of printed lines becomes more observable, changing from colorless to increasingly deep blue. Moreover, it was found that for each ink formulation, the printed line width is uneven at a lower voltage of $2000 \mathrm{~V}$. As the voltage increases to $2200 \mathrm{~V}$, the printed line width appears to become more even and smooth. This is probably due to insufficient electric field force generated by the lower voltage, resulting in an unstable jet, which in turn led to unstable printing. Clearly, the width of the printed lines is affected by applied voltage and $\mathrm{CuPc}$ particles content which were investigated respectively. Considering Figures 3(b-e) and (f), it can be seen that the width of the printed lines decreases as the applied voltage increases. This is mainly caused by the decreased jet diameter which has a positive effect on printing resolution. In addition, due to the addition of $\mathrm{CuPc}$ particles, the increasing viscosity of SR/CuPc ink is expected to hamper the spreading of the ink printed on the substrate and contribute to the narrow width of the printed lines. Owing to the higher printing resolution obtained under higher voltages, $2400 \mathrm{~V}$ was then chosen as an optimized voltage for printing different DE films, and some complex colored SR structures prepared using this voltage were depicted in the Figures S1. 

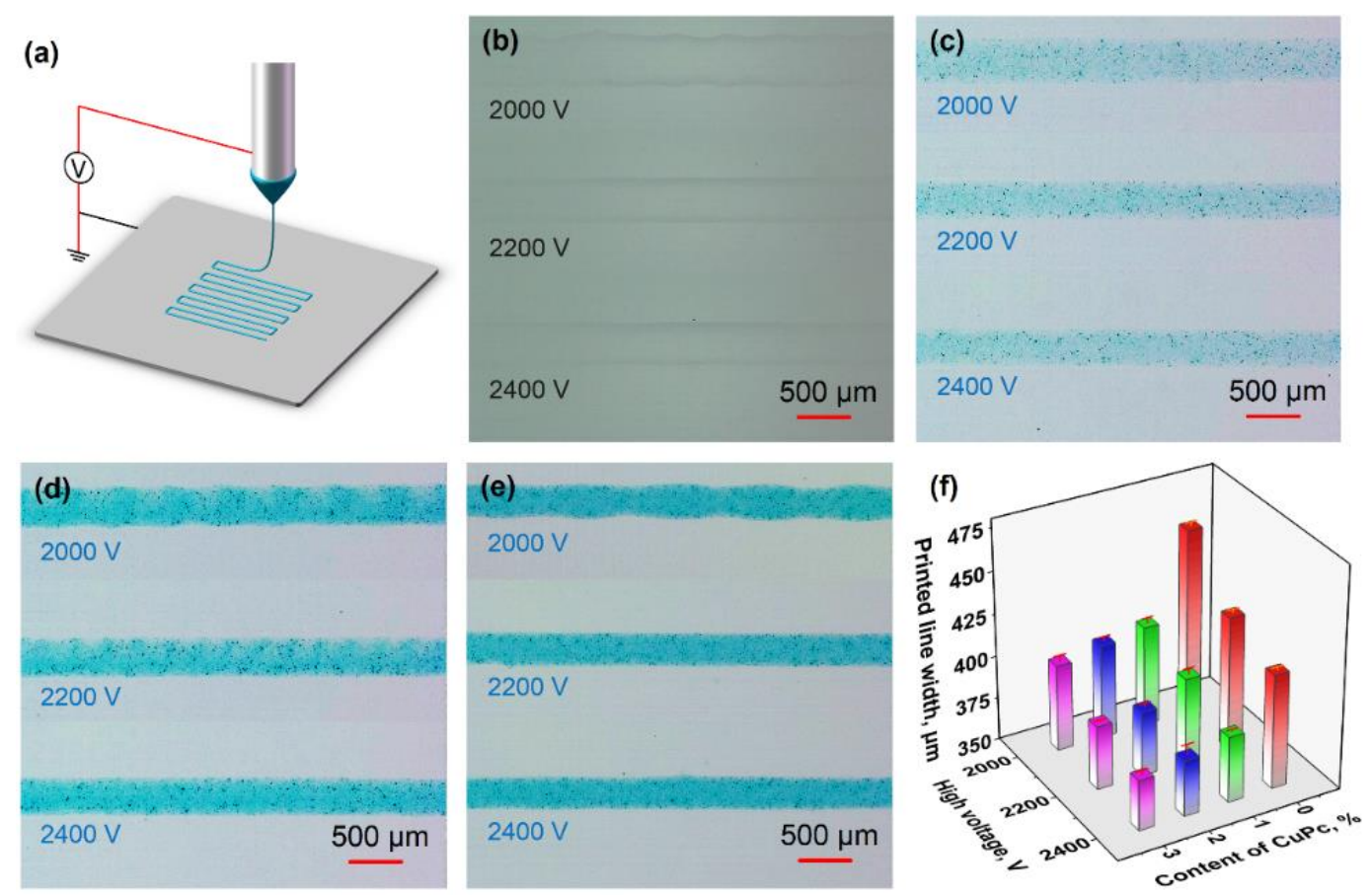

Figure 3 (a) Schematic of EHD 3D printing lines; Printed line morphologies of (b)

Pure SR; (c) SR/CuPc-1; (d) SR/CuPc-2 and (e) SR/CuPc-3; (f) Histogram of printed line diameter related to high voltage and content of $\mathrm{CuPc}$ particles.

Pure SR and SR/CuPc composite films were prepared by EHD 3D printing under the previously optimized conditions. In order to evaluate the dispersion of CuPc particles in the SR matrices, the surface morphologies of fractured pure SR and SR/CuPc composite films were observed. As can be seen from Figures 4(a-d), CuPc particles are not present in the fractured surface of pure SR (shown in Figure 4 (a)), and become gradually apparent and well dispersed in the fractured surface of the SR/CuPc composites with increasing content of $\mathrm{CuPc}$ particles from $1 \mathrm{wt} \%$ to $3 \mathrm{wt} \%$ (Figures 4(b-d)). Ultrasonic dispersion was introduced to the EHD printing process, which helped to some extent increase the dispersibility of CuPc particles in the SR matrix [33]. Since Nitrogen $(\mathrm{N})$ only exists in CuPc particles rather than in the SR, the distribution of CuPc particles in the SR matrices can be revealed by observing the distribution of $\mathrm{N}$ using EDX mapping. As shown in Figure 4 (e-h), a small amount of element $\mathrm{N}$ which is noise can be seen in the pure SR sample while there are uniform distributions of $\mathrm{N}$ 
element in all of the SR/CuPc composite films, indicating good dispersion of $\mathrm{CuPc}$ particles throughout the SR matrices.

The surface profiles of pure SR and SR/CuPc composite films were also characterized by using an ultra-depth 3D digital microscope. As can be seen from Figures 4 (i-1), the surface roughness of a typical printed pure SR membrane is about $15.94 \mu \mathrm{m}$, while the values of SR/CuPc-1, SR/CuPc-2, SR/CuPc-3 reach to about $18.92 \mu \mathrm{m}, 20.74 \mu \mathrm{m}$ and $24.50 \mu \mathrm{m}$ respectively, indicating that the addition of $\mathrm{CuPc}$ particles leads to a rougher surface of those resulted SR/CuPc composite films. This is most likely due to the fact that the increased viscosity of the inks caused by the addition of $\mathrm{CuPc}$ particles favors the gap formation of between adjacent lines of the printed patterns from completely.
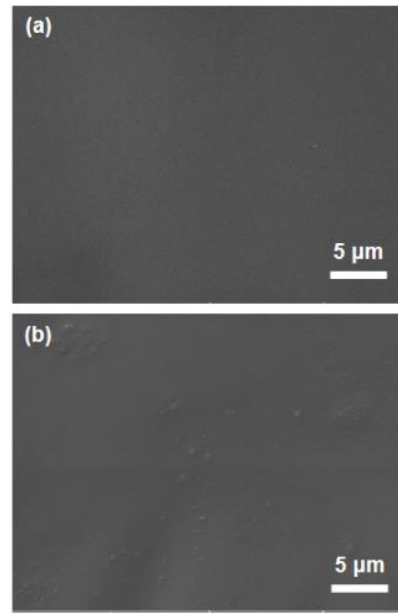

(c)

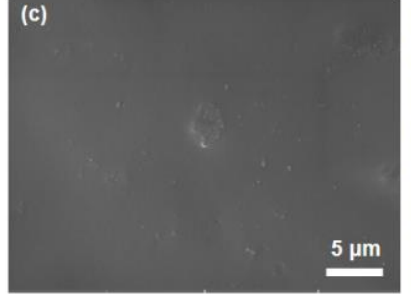

(d)

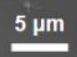

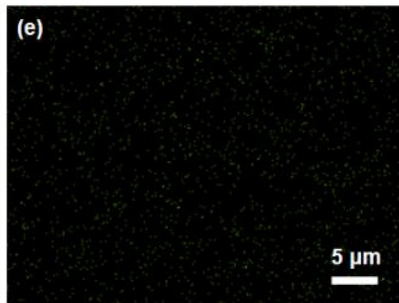

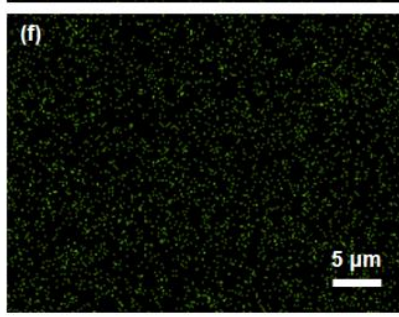

(g)

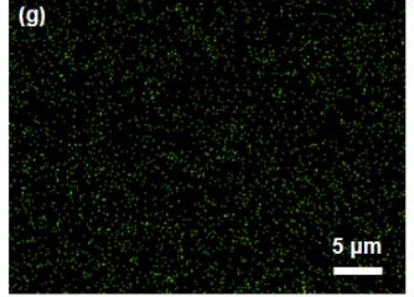

(h)

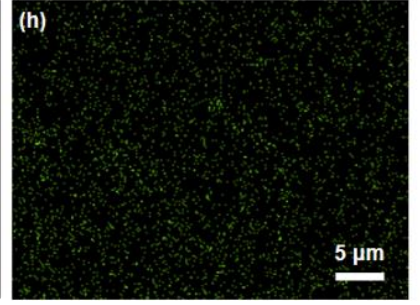

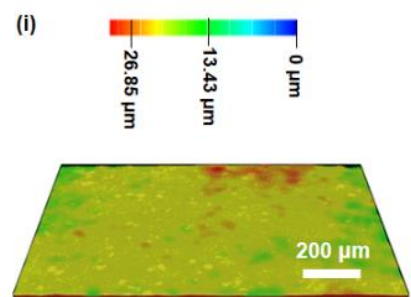

(j)
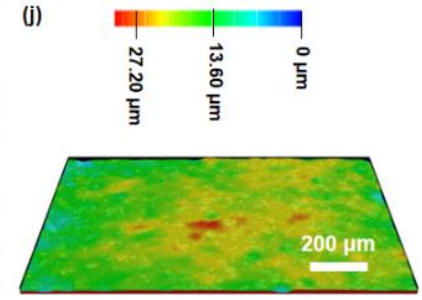

(k)

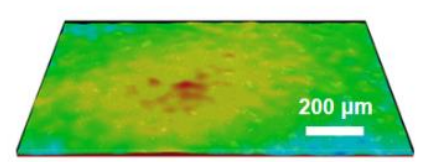

(I)
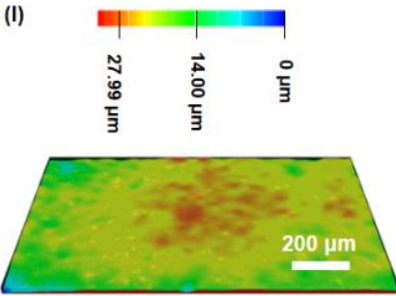

Figure 4 Surface morphologies of fractured SR and SR/CuPc films: (a) Pure SR;

(b) SR/CuPc-1; (c) SR/CuPc-2; (d) SR/CuPc-3; EDX mapping of the element of $\mathrm{N}$ in: 
(e) Pure SR; (f) SR/CuPc-1; (g) SR/CuPc-2 and (h) SR/CuPc-3; Ultra-depth 3D map of: (i) Pure SR; (j) SR/CuPc-1; (k) SR/CuPc-2 and (1) SR/CuPc-3.

Mechanical properties of EHD printed pure SR and SR/CuPc composite films are important considering their practical application. Typical stress-strain curves and the elastic modulus-strain curves (from $0 \%$ to $500 \%$ ) for SR and SR/CuPc composite films are shown in Figures 5(a) and (b), respectively. The tensile strength, elongation at break, elastic modulus and crosslink density are presented in Figure 5(c). It can be observed from Figure 5(a) that the pure SR film exhibits a low tensile strength of $1.54 \pm 0.61$ $\mathrm{MPa}$. After blending with $\mathrm{CuPc}$ particles, the tensile strength of SR/CuPc-3 composites increases to $4.16 \pm 0.91 \mathrm{MPa}$, approximately 2.7 times larger than that of the pure SR. This is mainly attributed to the reinforcing effect of CuPc particles [9]. Concurrently, the elongation at break of SR/CuPc composite films also increases with the increasing $\mathrm{CuPc}$ content. The elongation at break for pure SR is about $1309 \pm 44 \%$ and reaches $1526 \pm 29 \%$ for SR/CuPc-3 composite film. This could be ascribed to the decrease in the crosslink density enhancing the flexibility of the films $[35,36]$. As shown in Figure 5 (c), the crosslink density of SR films decreases from $1.68 \times 10^{-4} \mathrm{~mol} / \mathrm{cm}^{3}$ to $1.11 \times 10^{-4}$ $\mathrm{mol} / \mathrm{cm}^{3}$ as the content of CuPc increases from 0 to $3 \mathrm{wt} \%$. 

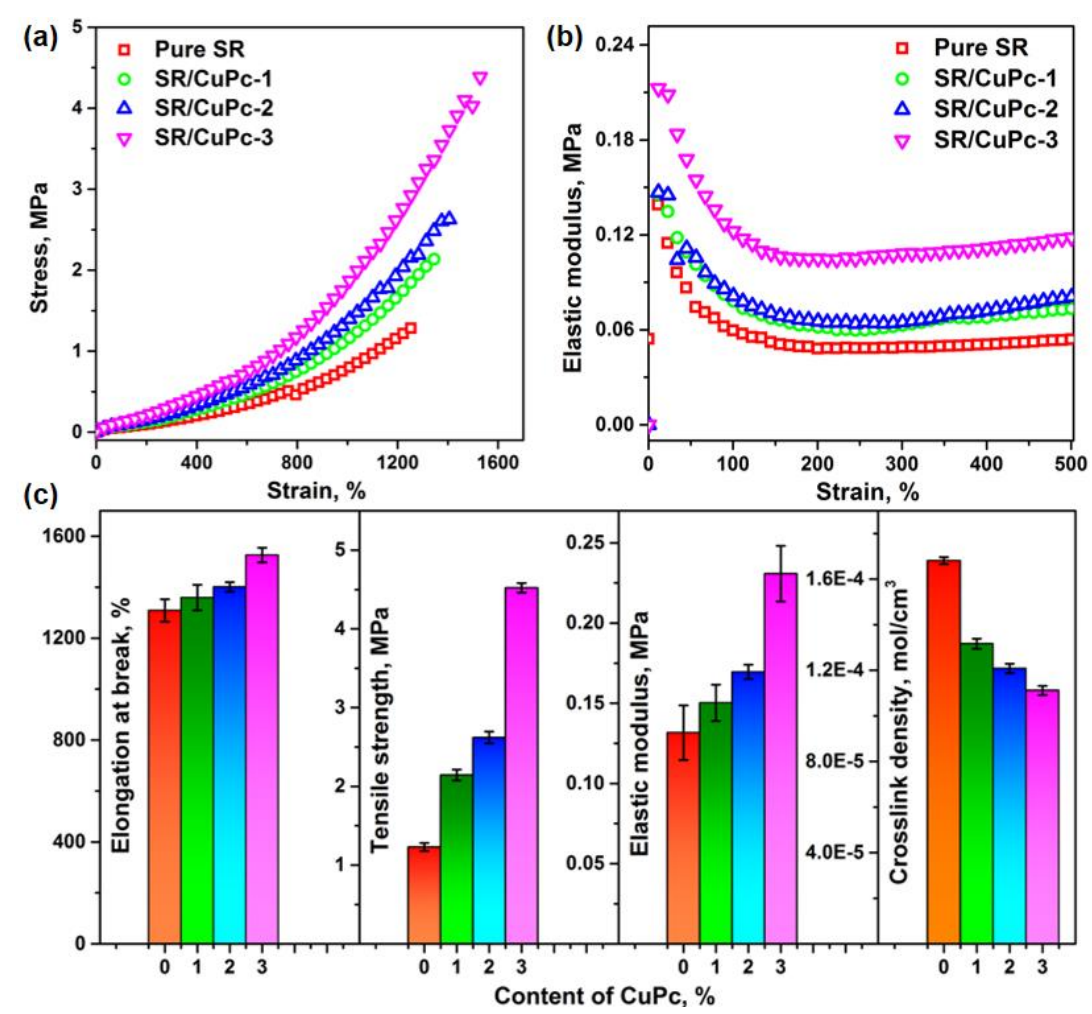

Figure 5 Mechanical properties of SR and SR/CuPc composites: (a) Stress-strain curves; (b) Elastic modulus-strain curves; (c) Histogram of elongation at break, tensile strength and elastic modulus changes with content of $\mathrm{CuPc}$.

As indicated in Figures 5(b) and (c), the elastic modulus of pure SR is $0.132 \pm 0.017$ $\mathrm{MPa}$, while that of SR/CuPc composites containing $1 \mathrm{wt} \% \mathrm{CuPc}, 2 \mathrm{wt} \% \mathrm{CuPc}$ and 3 $\mathrm{wt} \% \mathrm{CuPc}$ particles is $0.150 \pm 0.011 \mathrm{MPa}, 0.170 \pm 0.004 \mathrm{MPa}$ and $0.231 \pm 0.017 \mathrm{MPa}$, respectively. The change of this property can be explained by the fact that the addition of $\mathrm{CuPc}$ particles favors the formation of filler networks, which both disrupting and restricting the mobility of SR molecular chains [37]. Figure 5(b) also demonstrates that there is a material specific minimum in elastic modulus at a strain of about $100 \%$, indicating that a small ratio of pre-stretching contributed to a reduction in composite's stiffness. When the strain is below $100 \%$, the entanglement of molecular chains was initially unraveled subjecting to the external force, leading to a decrease in elastic modulus. When the strain is above $100 \%$, an increase in intermolecular forces caused by the reorientation of molecular chains as the stretch ratio increased, resulting in an 
increased elastic modulus [10, 13].

The dielectric properties including the dielectric constant and the dielectric loss are the key factors affecting the electro actuated area strain of DEs. The dielectric constant and dielectric loss of pure SR and SR/CuPc composite films as a function of frequency ranging from $10 \mathrm{~Hz}$ to $10 \mathrm{MHz}$ at room temperature were investigated. As can be seen from the dielectric spectra in Figure 6(a), the dielectric constant of pure SR is about 2.96 at the lowest frequency of $10 \mathrm{~Hz}$, while the dielectric constants of SR/CuPc-1, $\mathrm{SR} / \mathrm{CuPc}-2$ and SR/CuPc-3 increase to 4.06, 4.12 and 5.52 respectively due to the high dielectric constant of $\mathrm{CuPc}$ contributed by the nomadic polarization [38]. Even after mixing with SR matrix, nomadic polarization of CuPc particles still played a vital role, resulting in an increase in the dielectric constant of the SR/CuPc composite films [39]. As illustrated in Figure 6(b), the dielectric loss of pure SR (0.0037) is lower than that of three SR/CuPc composite films at the frequency of $10 \mathrm{~Hz}$. This is because the CuPc particles present in $\mathrm{SR} / \mathrm{CuPc}$ composite films behaved as a semiconductor and facilitates the transfer of electric charges, resulting in an increase in dielectric loss in the polymer $[1,40]$. Furthermore, for the same material, at low frequencies $(10 \mathrm{~Hz}$ to $100 \mathrm{~Hz}$ ), the dielectric loss was relatively large due to the loss associated with ionic mobility [41]. The relationship between dielectric constant and strain is shown in Figure 6 (c). It can be seen that as the strain increases from $0 \%$ to $150 \%$, the dielectric constant of all $\mathrm{SR} / \mathrm{CuPc}$ composite films declines by $45 \%, 31 \%, 47 \%$ and $56 \%$ for pure SR, $\mathrm{SR} / \mathrm{CuPc}-1, \mathrm{SR} / \mathrm{CuPc}-2$ and SR/CuPc-3, respectively. This could be because the strained polymer chains result in less movement in their constituent polar segments, leading to lower polar segment reorientation [42]. Consequently, this indicates that although pre-stretching reduces the stiffness of SR films and improves electromechanical stability [43], the dielectric constant also concurrently decreases, 
which hinders the occurrence of large actuated area strains according to Equation 1.
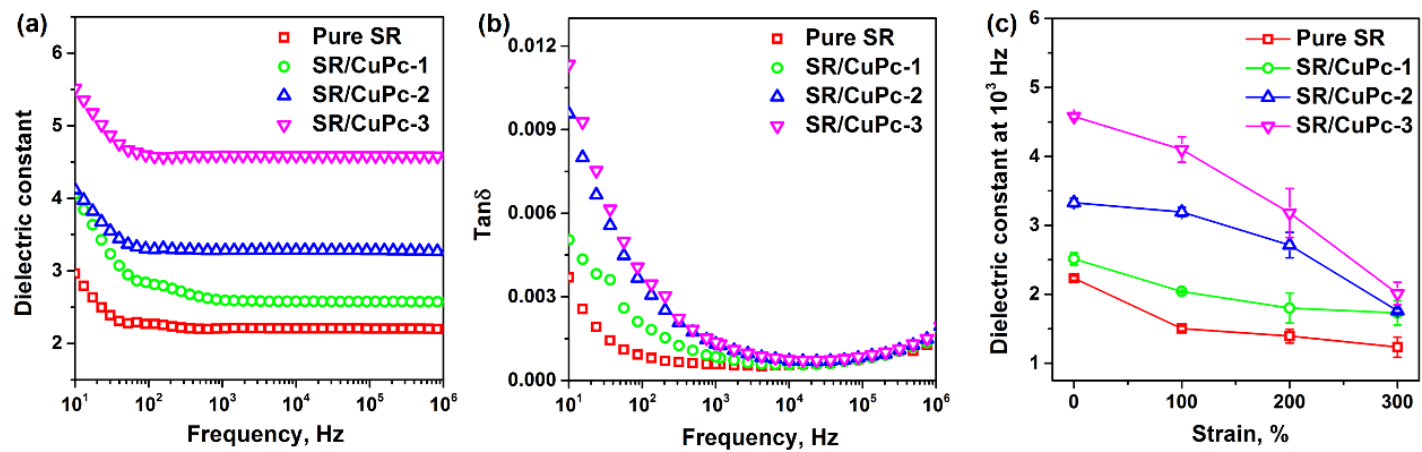

Figure 6 Dielectric spectra of EHD printed SR and SR/CuPc composites: (a) Plots of dielectric constant as a function of frequency; (b) Plots of dielectric loss tangent as a function of frequency; (c) plots of dielectric constant at $1 \mathrm{kHz}$ of SR and SR/CuPc composites against strain

Figure 7(a) depicts the electromechanical property testing system which consists of a high voltage power supply, a high-speed camera, a light device and an equi-biaxial sample clamp. The area was captured by the high-speed camera and measured using LabVIEW software in real time. The area strain $\left(s_{a}\right)$ was calculated from Equation 3.

$s_{a}=\left(A_{1}-A_{0}\right) / A_{0} \times 100 \%$

where $A_{0}$ is the initial area and $A_{1}$ is the actuated area.

Figure 7(b) present the electromechanical properties of pure SR and SR/CuPc composites. The pure SR presented an electro-induced area strain of about $16.17 \pm 0.55 \%$ at a breakdown electric field of approximately $50 \mathrm{~V} / \mu \mathrm{m}$. With the addition of $1 \mathrm{wt} \%$ $\mathrm{CuPc}$, the SR/CuPc-1 composite film exhibits an actuated area strain of $18.07 \pm 0.85 \%$ at an applied electric field of $43.5 \mathrm{~V} / \mu \mathrm{m}$. Furthermore, the SR/CuPc composite film containing $2 \mathrm{wt} \% \mathrm{CuPc}$ displays a maximum area strain of about $23.83 \pm 0.75 \%$, actuating at breakdown electric field of approximately $40.5 \mathrm{~V} / \mu \mathrm{m}$, while under a breakdown electric field of $39.4 \mathrm{~V} / \mu \mathrm{m}$, the SR/CuPc-3 DE performs the largest area strain of $25.5 \pm 0.72 \%$ which is much higher than that of inkjet printed silicone 
elastomer (6.1\%) [22]. In addition, as shown in Figure 7(c), with the increase in CuPc content, the maximum actuated area strains increase. This is ascribed to the higher ratio of dielectric constant to elastic modulus, which is called the electromechanical sensitivity $\left(\varepsilon_{r} / Y\right)$ [44]. Due to the addition of dielectric CuPc particles, SR/CuPc composite DEs have higher dielectric constant $\left(\varepsilon_{r}\right)$ and are therefore subjected to larger Maxwell stresses, facilitating higher electro-induced area strain [45]. Meanwhile, the addition of $\mathrm{CuPc}$ particles increases the modulus $(Y)$ of $\mathrm{SR} / \mathrm{CuPc}$ composite DEs and therefore hinder high electro-induced area strain. However, the prominence of the dielectric constant results in a considerable increase in $\varepsilon_{r} / Y$ from 16.7 to 20.0 , which can be seen in Figure 7(d). Furthermore, as can be observed from Figure 7(b), the breakdown electric field strength of $\mathrm{SR} / \mathrm{CuPc}$ composite films diminishes with an increase in the content of $\mathrm{CuPc}$. This was probably due to the fact that the agglomeration of $\mathrm{CuPc}$ particles improved the electrical conductivity, resulting in the composite film to be subject to a large sustaining dielectric loss [46].
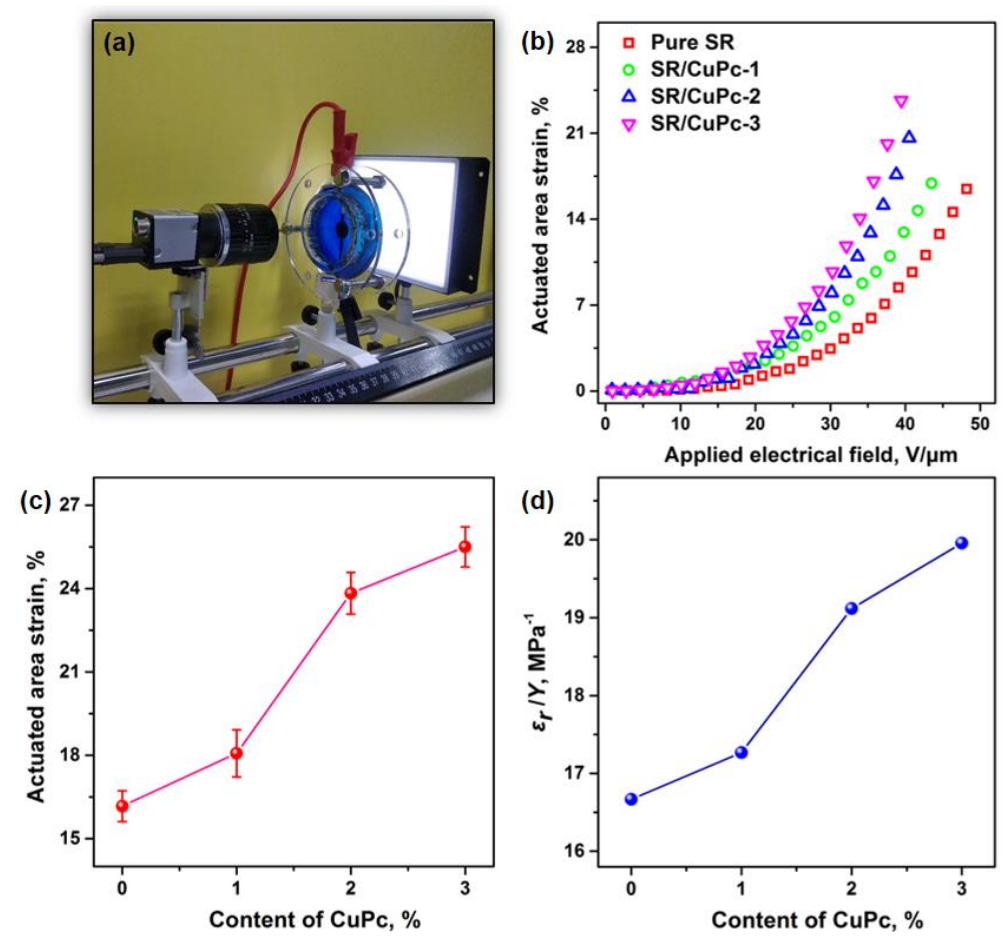

Figure 7 (a) Setup photograph of the electromechanical property testing system; (b) 
Plot of actuated area strain-applied electric filed curves of pure SR and SR/CuPc composite films; (c) Plot of actuated area strain as a function of the content of $\mathrm{CuPc}$ (d) Plot of electromechanical sensitivity as a function of $\mathrm{CuPc}$ content.

In order to evaluate the dynamic electromechanical properties of pure SR film and SR/CuPc-3 composite film, 100 cycles of electric field loading were conducted in a range between 0 and a maximum applied voltage of $3700 \mathrm{~V}$ (the highest electrical field achieved was $34.0 \mathrm{~V} / \mu \mathrm{m}$ ), with stepped voltage of $400 \mathrm{~V}$ per $3 \mathrm{~s}$. As shown in Figure 8 (a), pure SR film exhibits stable working behavior over 100 cycles of electric field loading with an actuated area strain of about $7.5 \%$. Figure $8(\mathrm{~b})$ demonstrates that although the actuated area strain of SR/CuPc-3, which has an overall good stability, gradually shifts to higher values with the increasing cycle number. For example, the actuated area strain at the first five cycles is about $13.4 \%$, while that at the last five cycles increases to about $14.1 \%$. An explanation for this could be that under the electrostatic force the deformation hysteresis during the compression in thickness caused an increase in the energy loss of SR/CuPc-3 composite film [10].
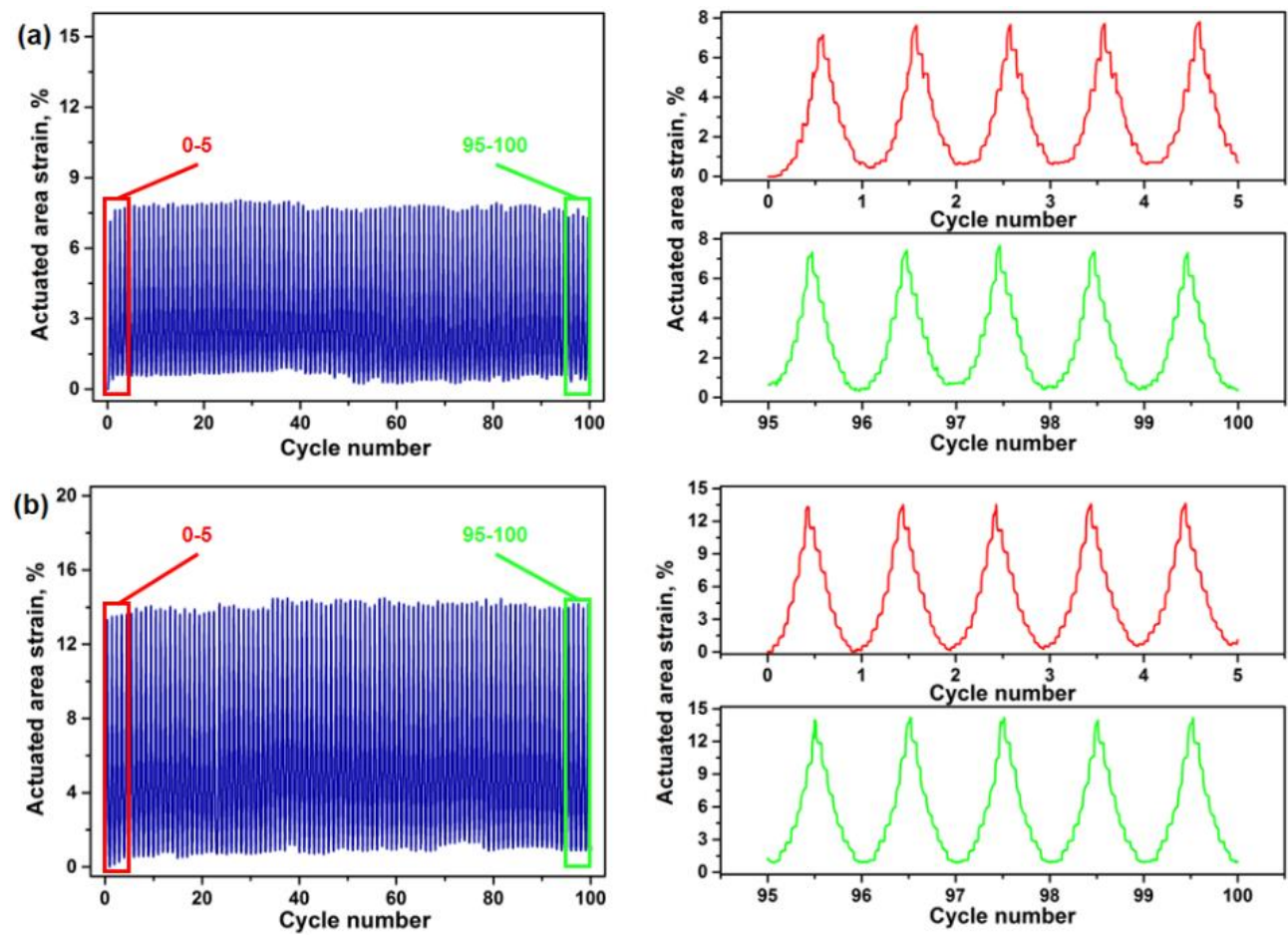
Figure 8 The dynamic electromechanical properties of (a) pure SR and (b) SR/CuPc3 under cyclic electric field loading at $34.0 \mathrm{~V} / \mu \mathrm{m}$.

\section{Conclusion}

In this work, a high-performance SR/CuPc DE film was successfully prepared by utilizing EHD 3D printing, which possessed the advantages of being fully automated, exhibiting flexibility and a high printing resolution. In order to obtain good printing resolution, higher voltages were required to decrease the jet diameter. Based on this, the optimal printed voltage of $2400 \mathrm{~V}$ was determined. However, it should be noted that the printed line width was much larger than the jet diameter at a constant voltage, which was ascribed to the fluidity of the ink. This situation can be improved by controlling the ink viscosity or by changing the matrix material to a thermal plastic elastomer. Additionally, the dielectric CuPc particles demonstrated good dispersibility in the SR matrix when using line by line EHD printing technology. For DEs prepared in this work, the obtained SR/CuPc-3 film possessed a high dielectric constant of 5.52, while delivering a high actuated area strain of $23.7 \%$ under an electric field of $39.4 \mathrm{~V} / \mu \mathrm{m}$. Moreover, SR/CuPc-3 showed a good stability under 100 cycles of electric field loading. EHD 3D printing provides a powerful and efficacious method for accurately fabricating high-performance DE films with different shapes.

\section{Acknowledgements}

The authors gratefully acknowledge the National Natural Science Foundation of China (Grant no. 51703108), the Shandong Provincial Natural Science Foundation, China (Grant no. ZR2017BEM042), the Shandong Provincial Key Research and Development Program, China (Grant no. 2019GGX102071 and 2018GGX108003) and the Shandong “Taishan Youth Scholar Program” for financial support.

\section{References}


[1] D. Yang, M. Tian, Y. Dong, H. Kang, D. Gong, L. Zhang, A high-performance dielectric elastomer consisting of bio-based polyester elastomer and titanium dioxide powder, Journal of Applied Physics 114(15) (2013) 154104.

[2] L. Jiang, Y. Zhou, Y. Wang, Z. Jiang, F. Zhou, S. Chen, J. Ma, Fabrication of Dielectric Elastomer Composites by Locking a Pre-Stretched Fibrous TPU Network in EVA, Materials 11(9) (2018) 1687.

[3] H. Imamura, K. Kadooka, M. Taya, A variable stiffness dielectric elastomer actuator based on electrostatic chucking, Soft Matter 13(18) (2017) 3440-3448.

[4] F. Carpi, G. Frediani, C. Gerboni, J. Gemignani, D.D. Rossi, Enabling variablestiffness hand rehabilitation orthoses with dielectric elastomer transducers, Medical Engineering \& Physics 36(2) (2014) 205-211.

[5] M. Follador, F. Tramacere, B. Mazzolai, Dielectric elastomer actuators for octopus inspired suction cups, Bioinspiration \& Biomimetics 9(4) (2014) 046002.

[6] R.E. Pelrine, R.D. Kornbluh, J.P. Joseph, Electrostriction of polymer dielectrics with compliant electrodes as a means of actuation, Sensors and Actuators A-physical 64(1) (1998) 77-85.

[7] B. Paul, P. Qibing, Advances in dielectric elastomers for actuators and artificial muscles, Macromolecular Rapid Communications 31(1) (2010) 10-36.

[8] L.J. Romasanta, M.A. Lopezmanchado, R. Verdejo, Increasing the performance of dielectric elastomer actuators: A review from the materials perspective, Progress in Polymer Science 51 (2015) 188-211.

[9] D. Yang, Y. Ni, X. Kong, Y. Wang, L. Zhang, A mussel-like inspired modification 
of $\mathrm{BaTiO}_{3}$ nanopartciles using catechol/polyamine co-deposition and silane grafting for high-performance dielectric elastomer composites, Composites Part B: Engineering $172(2019) 621-627$.

[10] L. Jiang, Y. Wang, S. Wen, Y. Zhou, J. Ma, S. Chen, S. Jerrams, Fabrication of dielectric elastomers with improved electromechanical properties using silicone rubber and walnut polyphenols modified dielectric particles, Materials \& Design 192 (2020) 108674.

[11] J. Wang, Z. Shi, X. Wang, X. Mai, R. Fan, H. Liu, X. Wang, Z. Guo, Enhancing dielectric performance of poly (vinylidene fluoride) nanocomposites via controlled distribution of carbon nanotubes and barium titanate nanoparticle, Engineered Science 4(14) (2018) 79-86.

[12] Q. Wang, J. Zhang, Z. Zhang, Y. Hao, K. Bi, Enhanced dielectric properties and energy storage density of PVDF nanocomposites by co-loading of $\mathrm{BaTiO}_{3}$ and $\mathrm{CoFe}_{2} \mathrm{O}_{4}$ nanoparticles, Advanced Composites and Hybrid Materials 3 (2020) 58-65.

[13] G. Gallone, F. Carpi, D.D. Rossi, G. Levita, A. Marchetti, Dielectric constant enhancement in a silicone elastomer filled with lead magnesium niobate-lead titanate, Materials Science \& Engineering C Biomimetic \& Supramolecular Systems C27(1) (2007) p.110-116.

[14] Y. Guo, N. Meng, J. Xu, K. Zhang, Q. Zhang, E. Pawlikowska, M. Szafran, F. Gao, Microstructure and dielectric properties of $\mathrm{Ba}_{0.6} \mathrm{Sr}_{0.4} \mathrm{TiO}_{3} /($ acrylonitrile-butadienestyrene)-poly (vinylidene fluoride) composites, Advanced Composites and Hybrid Materials 2(4) (2019) 681-689. 
[15] L. Shi, G. Song, P. Li, X. Li, D. Pan, Y. Huang, L. Ma, Z. Guo, Enhancing interfacial performance of epoxy resin composites via in-situ nucleophilic addition polymerization modification of carbon fibers with hyperbranched polyimidazole, Composites Science and Technology 201 (2020) 108522.

[16] J.-W. Wang, Y. Wang, F. Wang, S.-Q. Li, J. Xiao, Q.-D. Shen, A large enhancement in dielectric properties of poly (vinylidene fluoride) based all-organic nanocomposite, Polymer 50(2) (2009) 679-684.

[17] L. Maffli, S. Rosset, M. Ghilardi, F. Carpi, H. Shea, Ultrafast All-Polymer Electrically Tunable Silicone Lenses, Advanced Functional Materials 25(11) (2020) $1656-1665$.

[18] P. Lotz, M. Matysek, H.F. Schlaak, Fabrication and Application of Miniaturized Dielectric Elastomer Stack Actuators, IEEE/ASME Transactions on Mechatronics 16(1) (2011) p.58-66.

[19] O.A. Araromi, A.T. Conn, C.S. Ling, J. Rossiter, R. Vaidyanathan, S.C. Burgess, Spray deposited multilayered dielectric elastomer actuators, Sensors and Actuators Aphysical 167(2) (2011) 459-467.

[20] X. Wang, M. Jiang, Z. Zhou, J. Gou, D. Hui, 3D printing of polymer matrix composites: A review and prospective, Composites Part B: Engineering 110 (2017) $442-458$.

[21] M. Vaezi, H. Seitz, S. Yang, A review on 3D micro-additive manufacturing technologies, The International Journal of Advanced Manufacturing Technology 67(5) (2013) 1721-1754. 
[22] D. Mccoul, S. Rosset, S. Schlatter, H. Shea, Inkjet 3D printing of UV and thermal cure silicone elastomers for dielectric elastomer actuators, Smart Materials and Structures 26(12) (2017) 125022.

[23] A. Lee, H. Jin, H. Dang, K.H. Choi, K.H. Ahn, Optimization of Experimental Parameters To Determine the Jetting Regimes in Electrohydrodynamic Printing, Langmuir 29(44) (2013) 13630-13639.

[24] M.S. Onses, C. Song, L. Williamson, E. Sutanto, P.M. Ferreira, A.G. Alleyne, P.F. Nealey, H. Ahn, J.A. Rogers, Hierarchical patterns of three-dimensional blockcopolymer films formed by electrohydrodynamic jet printing and self-assembly, Nature Nanotechnology 8(9) (2013) 667-675.

[25] I. Liashenko, J. Rosell-Llompart, A. Cabot, Ultrafast 3D printing with submicrometer features using electrostatic jet deflection, Nature Communications 11(1) (2020) $1-9$.

[26] J. Park, M. Hardy, S.J. Kang, K. Barton, K. Adair, D.K. Mukhopadhyay, C.Y. Lee, M.S. Strano, A.G. Alleyne, J.G. Georgiadis, High-resolution electrohydrodynamic jet printing, Nature Materials 6(10) (2007) 782-789.

[27] G. Mathew, J.M. Rhee, C. Nah, D.J. Leo, Effects of silicone rubber on properties of dielectric acrylate elastomer actuator, Polymer Engineering \& Science 46(10) (2006).1455-1460.

[28] G. Zhang, H. Lan, L. Qian, J. Zhao, F. Wang, A Microscale 3D Printing Based on the Electric-Field-Driven Jet, 3D Printing and Additive Manufacturing 7(1) (2020) 3744. 
[29] S.-Y. Kim, Y. Kim, J. Park, J. Hwang, Design and evaluation of single nozzle with a non-conductive tip for reducing applied voltage and pattern width in electrohydrodynamic jet printing (EHDP), Journal of Micromechanics and Microengineering 20(5) (2010) 055009.

[30] D. Wang, X. Zhao, Y. Lin, J. Liang, T. Ren, Z. Liu, J. Li, Nanoscale coaxial focused electrohydrodynamic jet printing, Nanoscale 10(21) (2018) 9867-9879.

[31] J.L. Li, EHD sprayings induced by the pulsed voltage superimposed to a bias voltage, Journal of Electrostatics 65(12) (2007) 750-757.

[32] H. Wang, H. Kong, J. Zheng, H. Peng, C. Cao, Y. Qi, K. Fang, W. Chen, Systematically Exploring Molecular Aggregation and Its Impact on Surface Tension and Viscosity in High Concentration Solutions, Molecules 25(7) (2020) 1588.

[33] S.H. Hashimdeen, A prototype for 3D electrohydrodynamic printing, UCL (University College London), 2016.

[34] H. Lee, B. Seong, J. Kim, Y. Jang, D. Byun, Direct Alignment and Patterning of Silver Nanowires by Electrohydrodynamic Jet Printing, Small 10(19) (2014) 39183922.

[35] D. Yang, M. Tian, D. Li, W. Wang, F. Ge, L. Zhang, Enhanced dielectric properties and actuated strain of elastomer composites with dopamine-induced surface functionalization, Journal of Materials Chemistry 1(39) (2013) 12276-12284.

[36] X. Zhang, M. Wissler, B. Jaehne, R. Breonnimann, G. Kovacs, Effects of crosslinking, prestrain, and dielectric filler on the electromechanical response of a new silicone and comparison with acrylic elastomer, Proc Spie 5385 (2004) 78-86. 
[37] D. Yang, L. Zhang, N. Ning, D. Li, Z. Wang, T. Nishi, K. Ito, M. Tian, Large increase in actuated strain of HNBR dielectric elastomer by controlling molecular interaction and dielectric filler network, RSC Advances 3(44) (2013) 21896-21904.

[38] P.S. Vijayakumar, H.A. Pohl, Giant polarization in stable polymeric dielectrics, Journal of Polymer Science Part B 22(8) (1984) 1439-1452.

[39] H. Xu, Y. Bai, V. Bharti, Z.Y. Cheng, High dielectric constant composites based on metallophthalocyanine oligomer and poly(vinylidene fluoride-trifluoroethylene) copolymer, Journal of Applied Polymer Science 82(1) (2001) 70-75.

[40] V. Bobnar, A. Levstik, C. Huang, Q.M. Zhang, Dielectric Properties and Charge Transport in All-Organic Relaxorlike CuPc-P(VDF-TrFE-CFE) Composite and its Constituents, Ferroelectrics 338(1) (2006) 107-116.

[41] J. Sebastian, E.T. Thachil, J.J. Mathen, J. Madhavan, P. Thomas, J. Philip, M.S. Jayalakshmy, S. Mahmud, G.P. Joseph, Enhancement in the Electrical and Thermal Properties of Ethylene Vinyl Acetate (EVA) Co-Polymer by Zinc Oxide Nanoparticles, Open Journal of Composite Materials 05(3) (2015) 79-91.

[42] G. Kofod, R.D. Kornbluh, R. Pelrine, P. Sommer-Larsen, Actuation response of polyacrylate dielectric elastomers, Journal of Intelligent Material Systems and Structures 14(12) (2003) 787-793.

[43] B. Li, H. Chen, J. Qiang, S. Hu, Z. Zhu, Y. Wang, Effect of mechanical pre-stretch on the stabilization of dielectric elastomer actuation, Journal of Physics D: Applied Physics 44(15) (2011) 155301.

[44] D. Yang, L. Zhang, H. Liu, Y. Dong, Y. Yu, M. Tian, Lead magnesium niobate- 
filled silicone dielectric elastomer with large actuated strain, Journal of Applied Polymer Science 125(3) (2012) 2196-2201.

[45] J.W. Wang, Q.D. Shen, C.Z. Yang, High Dielectric Constant Composite of P(VDF-TrFE) with Grafted Copper Phthalocyanine Oligomer, Macromolecules 37(6) (2004) 2294-2298.

[46] C. Tian, J. Qiu, K. Zhu, J. Wang, J. Li, Copper Phthalocyanine Oligomer Noncovalent Functionalized Graphene-Polyurethane Dielectric Elastomer Composites for Flexible Micro-Actuator, Soft Materials 13(4) (2015) 210-218. 